\title{
mmWave Measurement of RF Reflectors for 5G Green Communications
}

\author{
Tao Hong $\mathbb{D}^{1},{ }^{1}$ Jin Yao, ${ }^{1}$ Cong Liu $\mathbb{D},{ }^{1}$ and Fei Qi $\mathbb{D}^{2}$ \\ ${ }^{1}$ School of Electronics and Information Engineering, Beihang University, Haidian District, Beijing, China \\ ${ }^{2}$ China Telecom Corporation Limited Beijing Research Institute, Beijing, China
}

Correspondence should be addressed to Tao Hong; hongtao@buaa.edu.cn

Received 29 November 2017; Accepted 3 April 2018; Published 15 May 2018

Academic Editor: Michel Kadoch

Copyright (C) 2018 Tao Hong et al. This is an open access article distributed under the Creative Commons Attribution License, which permits unrestricted use, distribution, and reproduction in any medium, provided the original work is properly cited.

In recent years, with the energy consumption and environmental degradation, science and technology embarked on a path of sustainable development. In this situation, 5G green communication system has been widely used. This paper introduces the application of RF reflectors to $5 \mathrm{G}$ mmWave, where line-of-sight (LoS) blockage is a major hindrance for the coverage. In particular, we investigate the lab measurement of RF reflectors, which is a critical step from the theory to the practice. Furthermore, through the lab measurement, a 3D near-field range migration (RM) imaging algorithm for MIMO array configuration is proposed, and the sampling scheme is improved to save the computation time while providing high-quality images.

\section{Introduction}

In $5 \mathrm{G}$ communications, energy efficiency has become a matter of prime importance for wireless networks. A great deal of research has been done in the past to reduce energy consumption. Resource allocation, heterogeneous network deployment, transmission scheme optimization, and the development of energy-efficient algorithms have become the focus of research. Small Cell deployment is a widely accepted strategy in the industry to achieve various performance and efficiency indicators for the future $5 \mathrm{G}$ system. However, the deployment of Small Cell base stations generally has problems in maintenance and insufficient site resources. This paper proposes a method of using the RF reflector, reflecting only the existing wave, without additional energy consumption, reducing land occupation, saving maintenance resources, and having higher commercial utilization value.

Compared to traditional active repeaters, the universal interest candidates of passive repeaters (PRs) have been inevitably utilized in massive multiple-input-multiple-output (MIMO) system, with a multitude of advantages of lower cost of maintenance, manufacturing, and operation.

In order to experimentally discuss the information capacity of MIMO in multipath environment, a PR includes a power combiner, a planar Yagi-Uda antenna, and a fourelement folded-patch antenna (FPA) array $[1,2]$. The discussion results demonstrate that the performance of broad-angle scattering and polarization transition can be implemented. Simultaneously, the received power and channel capacity of the system gain an achievement that the four-unit PR can improve the propagation channel in wireless access system.

Existing research proposes a system of MIMO channel operation with tunable passive repeater [3]. The PR has a multitude of antennas, and a phase-shifter function exists between these antennas. The elements of the PR can be controlled so that a maximized MIMO channel capacity can be gained [4]. Experimental results can reveal the effectiveness of the proposed passive repeater.

Reflect-array antenna is a flat low-profile reflector including a planar array of microstrip patch elements to reflect the special beam direction and shape with certain tuning when primary source illuminates it $[5,6]$. Compared with conventional parabolic reflector antenna, the planar reflectarray plays a more significant role in the MIMO system for the ability to surface-mount the reflect-array in virtue of its convenient deployment, lower cost of manufacturing, small volume, low mass, and so forth. 


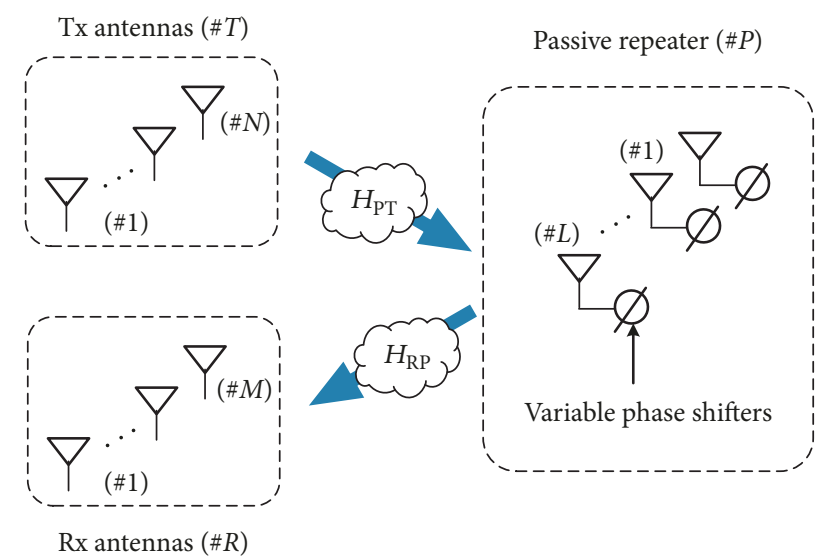

Figure 1: The channel transmission procedure with passive repeater.

Reflectors can be used commonly to improve the propagation channel conditions, and the ability is exhibited prominently in inherently bad-conditioned environment. Most researches show that PR can improve the MIMO system performance because MIMO has multiple streams to transfer, requiring multiple paths [7]. In terms of passive repeater, there is no amplifier and there is no oscillator in the repeater.

The use of reflector in MIMO system has opened up a new line of thinking that propagation channel can be changed intentionally, for example, moving objects. To pursue higher performance gain and drive greater system operational efficiency, a more active method is required.

The mmWave communications utilize the $30-300 \mathrm{GHz}$ frequency band with rich spectrum resources for multigigabit transmissions, which is one of the most promising technologies for $5 \mathrm{G}[8,9]$. In $[10-12]$, it is shown that, by using a highly oriented antenna array, the millimeterwave band can be allocated to cellular communications. Higher frequencies lead to higher bandwidth. Advanced RF beamforming techniques using high-gain advanced antennas at millimeter-wave frequencies and MIMO digital beamforming technology support the development of RF reflectors $[13,14]$. Therefore, the study of this paper is based on the millimeter-wave band.

The mmWave indoor propagation simulation for real-life office environments was presented by using 3D shootingand-bouncing ray tracing and measurement in paper. What is more, the non-line-of-sight (NLoS) channel environment is improved by devising the new passive repeaters in mmWave frequency bands, and at the same time the repeaters are embodied in the ray tracing procedure.

Currently, Pozar et al. proposed that a broadband reflectarray can be regarded as a PR to solve the problem of blind areas [15]. Nevertheless, if a very large scattering angle was tested in the case of a physical limitation of the reflect-array, the aperture efficiency of the reflect-array was reduced to a lower rank greatly.

\section{Basics of Reflect-Array as 5G Repeater}

2.1. Passive Repeater Principle. Figure 1 demonstrates the proposed passive repeater. In this scheme, there are $N$ and $M$ antennas at the transmitter $(\# T)$ and receiver $(\# R)$, respectively. Assume that the signal on the direct path from the transmitter to the receiver is weak. The PR (\#P) locates among $L$ antennas. The channel matrices from $\# T$ to $\# P$ and from $\# P$ to $\# R$ are denoted by HPT and HRP, respectively.

The phase shift at the passive repeater is given by

$$
\Theta=\left(\begin{array}{ccc}
e^{j \theta_{1}} & & 0 \\
& \ddots & \\
0 & & e^{j \theta_{L}}
\end{array}\right) \text {; }
$$

the channel from \#T to \#R is denoted by

$$
H=H_{\mathrm{RP}} \Theta H_{\mathrm{PT}}
$$

where $\left[\theta_{1}, \ldots, \theta_{L}\right]$ describes the number of the phase shifts at $L$ antennas.

As mentioned above, the proposed tunable passive repeater scheme is quite feasible. When the number of antennas available is sufficient, the value of the phase shift is possible to be discrete and binary, for example, $[0,180]$ deg. Actually, some phase shift patterns are randomly assigned to $\mathrm{Rx}$, which can observe the throughput of the system. And Rx feeds back the best from all those patterns to the repeater. Even if the binary phase shifters are applied, a multiple of phase patterns $(2 L)$ exist. Some patterns have no occasion to be tested, for pretty good one is allowed and it does not have to be the best. With computational efficiency, genetic algorithm (GA) is so popular due to the readily available suboptimal solution. Thus, GA can ensure the maximum channel capacity and throughput by deciding which phase pattern is chosen.

Figure 2(a) illustrates that there are three kinds of path consisting of penetrated paths, diffracted paths, and reflected paths in NLoS area. As illustrated in Figure 2(c), all of these paths are used in ray tracing simulation. In these paths, a passive repeater is used and a reradiated path is added to solve the problem that these paths have large losses in NLoS path. Figure 2(b) illustrates a reradiated path generated by the passive repeater. The new procedure for the ray tracing simulation of passive repeater is shown in 


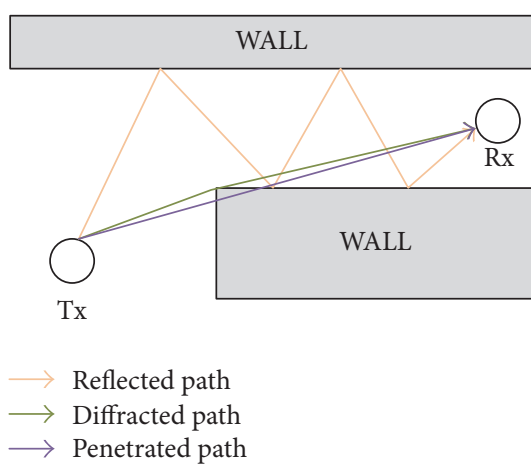

(a)

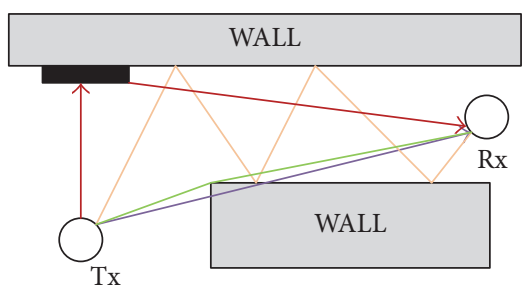

$\longrightarrow$ Reradiated path by passive repeater

$\longrightarrow$ Reflected path

$\longrightarrow$ Diffracted path

$\longrightarrow$ Penetrated path

(b)

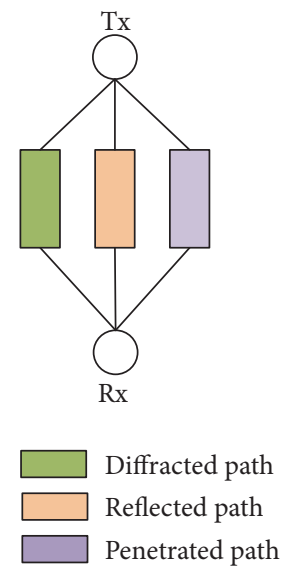

(c)

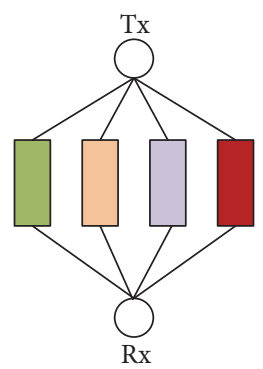

Diffracted path

Reflected path

Penetrated path

Direct path + Repeater pattern+ Reradiated path

(d)

FIGURE 2: Propagation procedure with or without passive repeater.

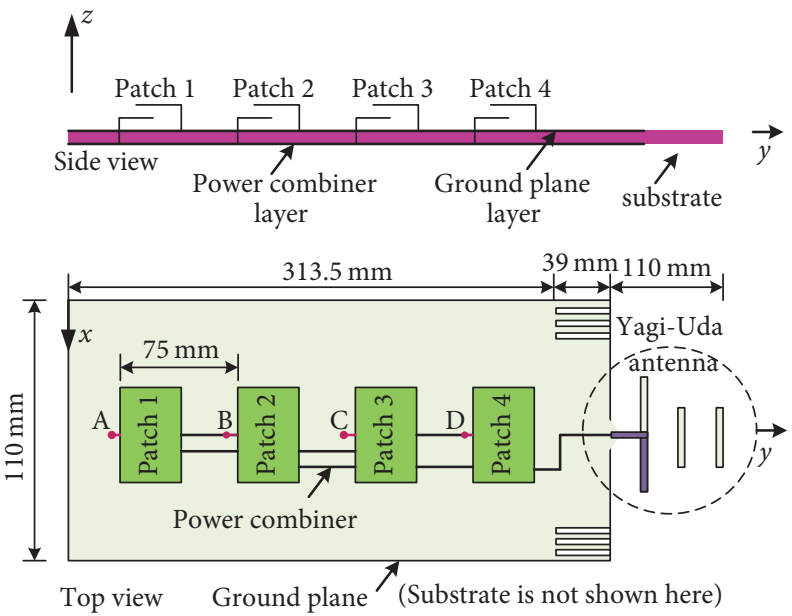

Figure 3: Configuration of one-unit PR.

Figure 2(d). Reradiated paths can be acquired by using the bistatic radar cross section (RCS) patterns and the receiving power of the passive repeater [9]. All of paths, consisting of direct paths, diffracted paths, penetrated paths, reflected paths, and reradiated paths, can be combined via postprocessing.
The configuration of the one-unit passive repeater is illustrated in Figure 3, consisting of a power combiner, a planar Yagi-Uda antenna, and a four-element FPA array. Because of their compact size, the FPA elements locate on the top side of the substrate, which are chosen the same as Set 1 in [2]. Balance between the antennas' size and gain 


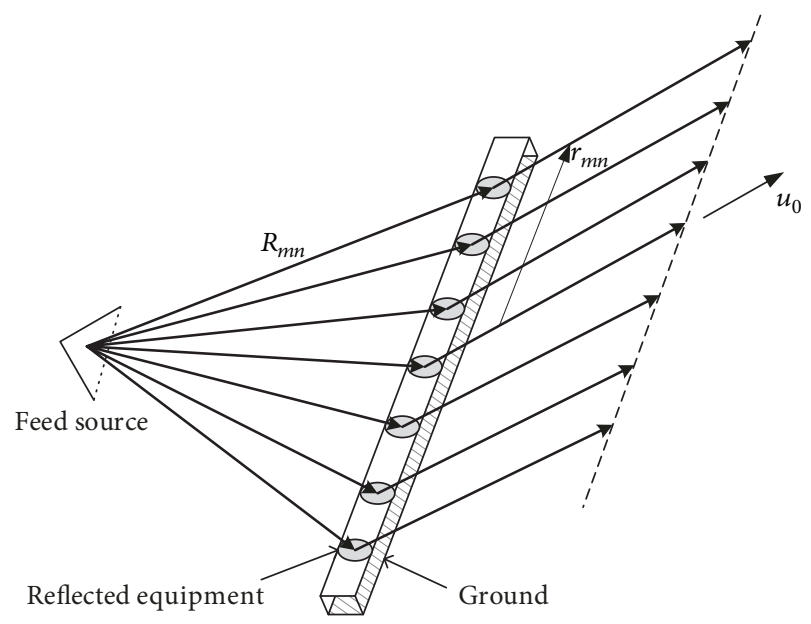

FIGURE 4: Configuration of microstrip reflect-array.

is a significant and versatile design. Therefore, when high gain is of main focus in industry and academia, other flat antenna types with slightly larger sizes can also be chosen to be satisfied.

The most common Yagi-Uda arrays are fabricated with one driven element and two directors, and the ground plane lies on 0.25 away from the patch and merely acts as a reflector [12]. In addition, the corrugated periodic ground layer with the appropriate parameters can improve the antenna gain by supporting or suppressing some of the sidelobes in the $\mathrm{H}$ plane. The bottom side power combiner is connected to the input port of the flat-bottomed antenna. The feed probe of the patch element passes through the hole in the ground plane and is connected to the input port of the power combiner.

In fact, the incident wave can point in any direction through the PR reflection. When receiving the FPA array, the received electromagnetic (EM) wave can be transmitted to the Yagi-Uda antenna [16]. Reference [5] revealed more details about the PR.

2.2. Reflect-Array Principle. Each element in the PR needs to be specifically designed to generate the beam in a specific direction by scattering the incident wave by appropriate phase compensation. Figure 4 illustrates the structure of a standard microstrip reflect-array. Because of effect from the reflected elements, the reradiated field was formed by the dipoles in a random direction.

$$
\begin{gathered}
E(u)=\sum_{m=1}^{M} \sum_{n=1}^{N} F\left(\vec{r}_{m n} \cdot \vec{r}_{f}\right) A\left(\vec{r}_{m n} \cdot \widehat{u}_{0}\right) A\left(\widehat{u} \cdot \widehat{u}_{0}\right) \\
\cdot \exp \left\{-j k_{0}\left[\left|\vec{r}_{m n}-\vec{r}_{f}\right|+\vec{r}_{m n} \cdot \widehat{u}\right]+j \phi_{m n}\right\},
\end{gathered}
$$

where $F$ is defined as the feed pattern function and $A$ is defined as the pattern function of the parasitic dipole element. The position vector for the mnth element is defined by $\vec{r}_{m n}$ and for the feed horn antenna is defined by $\vec{r}_{f}$. The desired main-beam pointing direction of the reflect-array is defined by $\widehat{u}_{0}$. The phase that is required for the scattered field from the $m n$th element is defined by $\phi_{m n}$.
In order for the aperture distribution to reach the desired direction, $\widehat{u}_{0}$, the condition is described by

$$
\phi_{m n}-k_{0}\left(R_{m n}+\vec{r}_{m n} \cdot \widehat{u}_{0}\right)=2 p \pi,
$$

$$
p=0, \pm 1, \pm 2, \ldots,
$$

where the distance from the mnth array element to the feed source is defined by $R_{m n}$; that is, $R_{m n}=\left|\vec{r}_{m n}-\vec{r}_{f}\right|$.

$k_{0}$ is the wave number, and the expression is $k_{0}=\omega_{0} / c$ with $\omega_{0}$ defined as the working frequency of the frequency selective surface (FSS) ground.

In wireless communications, it is a big deal for eliminating the blind spots of base station antennas in a complex street, high-building district, and many occlusions area. Typically, radio frequency $(\mathrm{RF})$ boosters have the ability to enlarge the cellular coverage area, while standard RF boosters have high cost of transceivers, power supplies, cables, and so forth and are limited in installation areas [17].

Because of the blockage of the building, the signal received for a part of users from BS is poor. Considering this point, the signal received for a part of users who are in the occlude areas can be enhanced via using a passive repeater as shown in Figure 5. The gray area and the green area represent the area occupied by the building and the area reflected by the passive repeater, respectively.

In the actual situation, the channel environment is timevarying and becomes relatively complex by moving objects, rough surfaces, or sharp edges of objects (such as cars, leaves, and lampposts). In complex streets, from the transmitter to the receiver in the wireless communication process, the frequency of the signal projected on the moving car will be offset, and the scattering phenomenon will affect the wireless communication, shown in Figure 6.

\section{3D Near-Field RM Imaging Algorithm}

In the MIMO configuration, the 3D image reconstruction process can be accomplished analogically on 2D imaging; that is, the backscattered data is coherently integrated over the 


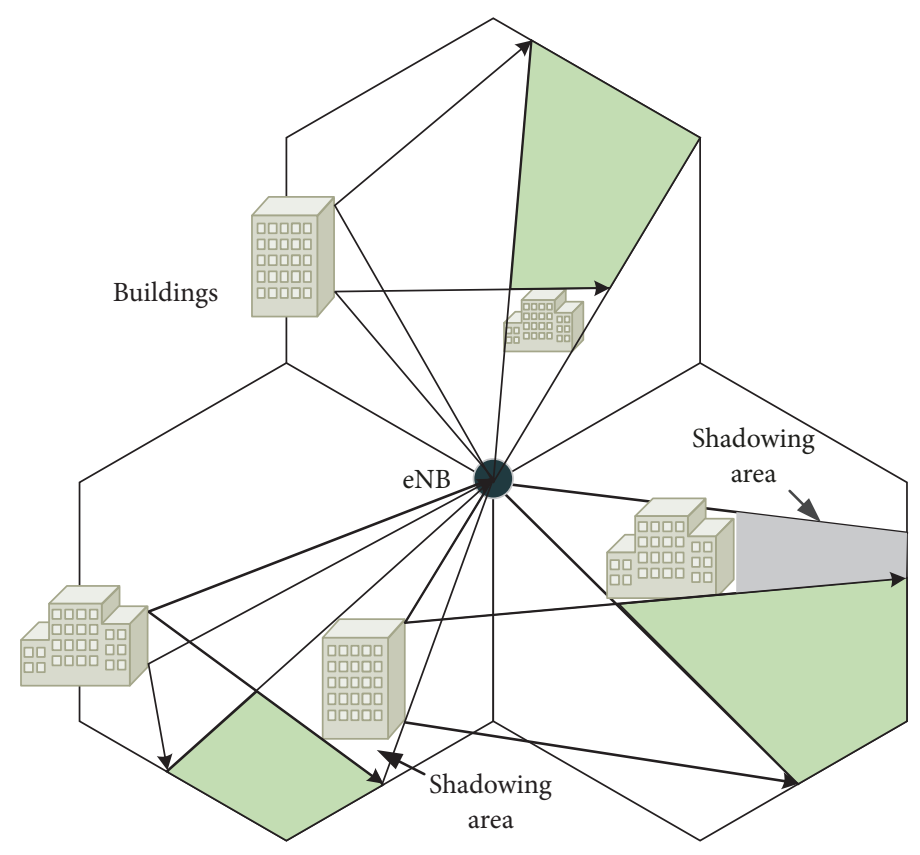

FIGURE 5: Reviving the signal of shadowing area.

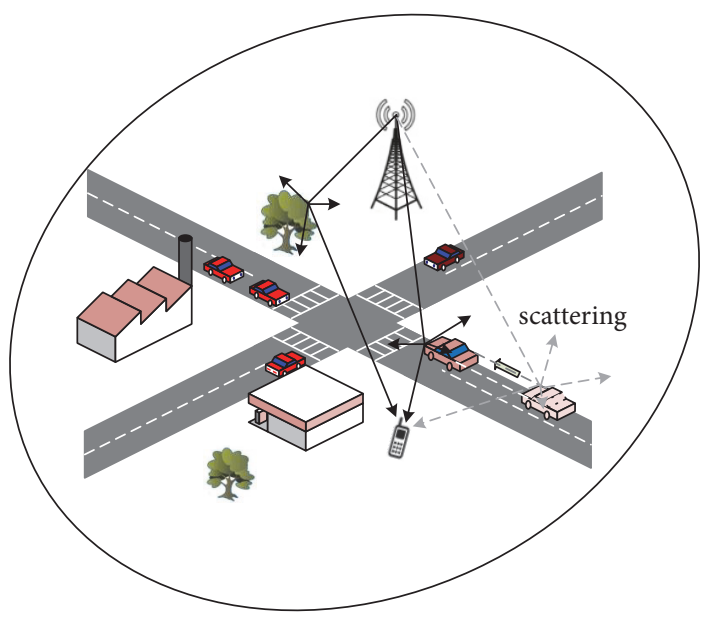

FIGURE 6: Scattering in complex channel environment.

two spatial coordinates of the 2D aperture and measured frequency band. The antenna array produces spherical waves in the near field. Considering the influence of spatial resolution and sampling interval, this paper uses time slot interpolation to determine a dedicated time and phase to obtain evenly spaced data [18].

The improved sampling method proposed in this paper effectively solves the problem that the traditional 3D imaging sampling time is too long and saves a lot of sampling time under the premise of reducing image distortion [19].

Figure 7 presents a 2D linear MIMO imaging geometry structure. In the wave field of the continuous wave, the MIMO array composed of the transmitting antenna and the receiving antenna illuminates the target plane located near the array aperture.
In the imaging process shown in Figure 7, the scanning range is $\left[L_{X}, L_{X}\right]$, the sampling interval is $\Delta X$, and the step frequency of the sampling point is $f$. The target position is set to $(X, Z)$; the transmitting antenna and the receiving antenna are located at $\left(X_{\mathrm{T}_{\mathrm{x}}}, 0\right),\left(X_{\mathrm{Rx}_{\mathrm{x}}}, 0\right)$, respectively, where the subscript in the formula represents the axis $x, y$. Therefore, the transmit and receive phase delays are $2 k R$, where $k=$ $2 \pi f / c, R=R_{\mathrm{TX}_{\mathrm{X}}}+R_{\mathrm{RX}_{\mathrm{X}}}, R_{\mathrm{T}_{\mathrm{X}}}=\sqrt{X-\left(X_{\mathrm{TX}}\right)^{2}+Z^{2}}$, and $R_{\mathrm{RX}_{\mathrm{X}}}=$ $\sqrt{X-\left(X_{\mathrm{Rx}}\right)^{2}+Z^{2}}$.

Let the received signal be $s\left(X_{\mathrm{Tx}}, X_{\mathrm{Rx}}, k\right)$, and the target reflectivity is $\sigma(X, Z)$. Consider the spreading loss; $s\left(X_{\mathrm{T}_{\mathrm{X}}}, X_{\mathrm{R}_{\mathrm{X}}}, k\right)$ can be expressed as

$$
s\left(X_{\mathrm{TX}_{\mathrm{X}}}, X_{\mathrm{Rx}}, k\right)=\frac{1}{4 \pi R_{\mathrm{T}_{\mathrm{X}}} R_{\mathrm{R}_{\mathrm{X}}}} \sigma(X, Z)
$$




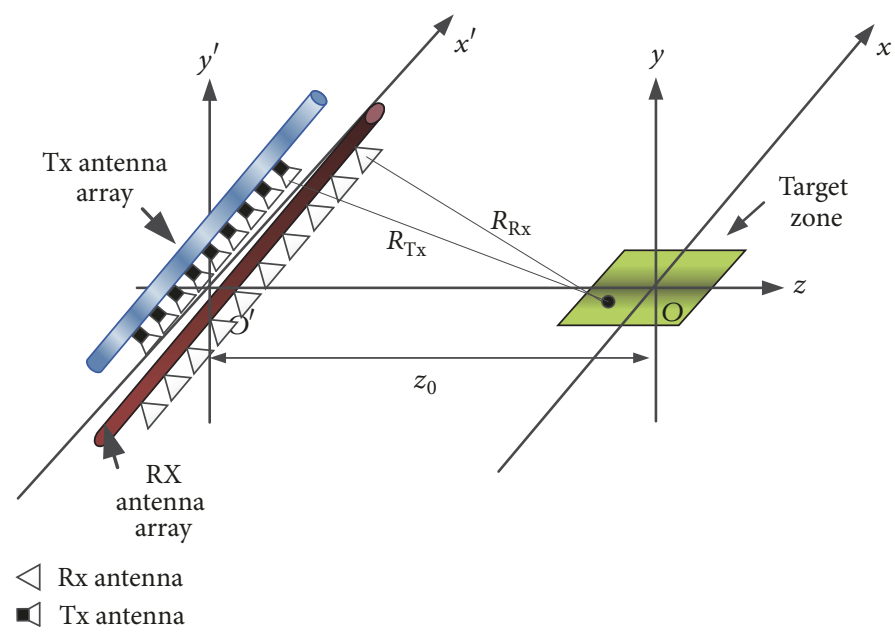

FIGURE 7: 2D linear MIMO imaging geometry structure.

$$
\cdot \exp \left(-j k\left(R_{\mathrm{Tx}_{\mathrm{x}}}+R_{\mathrm{R}_{\mathrm{X}}}\right)\right)
$$

where $R_{\mathrm{Tx}}$ represents the distance from the transmitter to the target and similarly $R_{\mathrm{Rx}}$ represents the receiver's distance.

The Fourier transform is performed on the received signal:

$$
\begin{aligned}
s\left(k_{X-T}, k_{X-R}, k\right)= & \frac{1}{4 \pi} \sigma(X, Z) \cdot F\left(k_{X-T}, k\right) \\
& \cdot F\left(k_{X-R}, k\right),
\end{aligned}
$$

where

$$
\begin{aligned}
F\left(k_{x-T}, k\right) & =\int \frac{\exp \left(-j k_{x-T} R_{\mathrm{T}_{\mathrm{x}}}\right)}{R_{\mathrm{T}_{\mathrm{x}}}} d X_{\mathrm{T}_{\mathrm{x}}} \\
& =\frac{j 2 \pi}{k_{z-T}} \exp \left(-j k_{z-T} \cdot Z-j k_{x-T} \cdot X\right) \\
F\left(k_{x-R}, k\right) & =\int \frac{\exp \left(-j k_{x-R} R_{\mathrm{R}_{\mathrm{x}}}\right)}{R_{\mathrm{R}_{\mathrm{x}}}} d X_{\mathrm{R}_{\mathrm{x}}} \\
& =\frac{j 2 \pi}{k_{z-R}} \exp \left(-j k_{z-R} \cdot Z-j k_{x-R} \cdot X\right) \\
k_{x} & =k_{x-T}+k_{x-R} \\
k_{z} & =\sqrt{k^{2}-k_{x-T}^{2}}+\sqrt{k^{2}-k_{x-R}^{2}} .
\end{aligned}
$$

The above analysis applies to the corresponding transceiver pair of a single scattering point; the following formula is used to represent the total received wave field:

$$
\begin{aligned}
& b\left(k_{x-T}, k_{x-R}, k\right)=\iint s\left(k_{x-T}, k_{x-R}, k\right) d x d z \\
& \quad=-\frac{\pi}{k_{z-T} k_{z-R}} \\
& \quad \cdot \iint \sigma(X, Z) \cdot \exp \left(-j k_{x} X-j k_{z} Z\right) d x d z .
\end{aligned}
$$

So the reflection rate of $2 \mathrm{D}$ imaging is calculated as

$$
\begin{aligned}
\sigma(X, Z) & =\iint \bar{b}\left(k_{X}, k_{Z}\right) e^{\left(j k_{x} X+j k_{z} Z\right)} d k_{x} d k_{z} \\
& =\text { ifft }\left\{\bar{b}\left(k_{X}, k_{Z}\right)\right\},
\end{aligned}
$$

where

$$
\bar{b}\left(k_{X}, k_{Z}\right)=-\frac{k_{x-T} k_{z-T}}{\pi} b\left(k_{x}, k_{z}\right)
$$

The above algorithmic model describes the specific process of 2D imaging. Similarly, a three-dimensional data array can be obtained for each pair of transceivers at each sampling point, which is easily generalized to the $3 \mathrm{D}$ range. Figure 8 shows a MIMO array 3D imaging geometry.

So the reflectivity map of $3 \mathrm{D}$ imaging is calculated as

$$
\begin{gathered}
\sigma(x, y, z)=\frac{1}{8 \pi^{3}} \iiint\left\{\iiint\left[s\left(x, y, k ; z_{0}\right) e^{\left(j k_{z} z_{0}\right)}\right]\right. \\
\left.\cdot \exp \left[-j\left(k_{x} x+k_{y} y+k_{z} z\right)\right] d x d y d z\right\} \\
\cdot \exp \left[-j\left(k_{x} x+k_{y} y+k_{z} z\right)\right] d k x d k y d k z
\end{gathered}
$$

The backscattered data set obtained during the imaging process is represented by $s\left(x, y, k ; z_{0}\right)$. Figure 9 is a complete flow diagram of 3D MIMO-RMA image reconstruction.

\section{Numerical Simulation and Spatial Sampling Scheme}

Figure 10 shows the target used in the numerical simulation. The target includes 27 scattering points and the distance between the target center and the antenna array is $0.75 \mathrm{~m}$. The area of the antenna array is $1 \mathrm{~m} \times 1 \mathrm{~m}$; the transmitting antenna and the receiving antenna are separated by $0.5 \mathrm{~cm}$. The frequency ranges from 8 to $12 \mathrm{GHz}$, sampling a total of 101 points with a step of $40 \mathrm{MHz}$. 


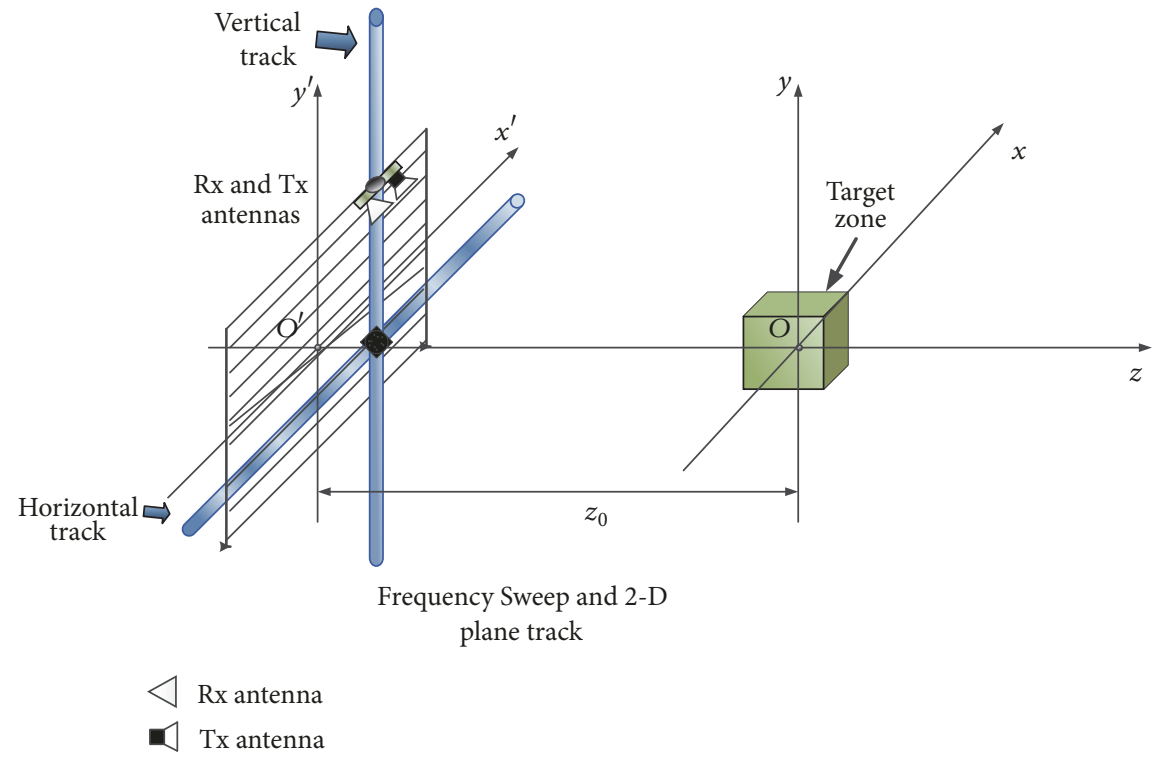

FIGURE 8: 3D MIMO array imaging geometry structure.

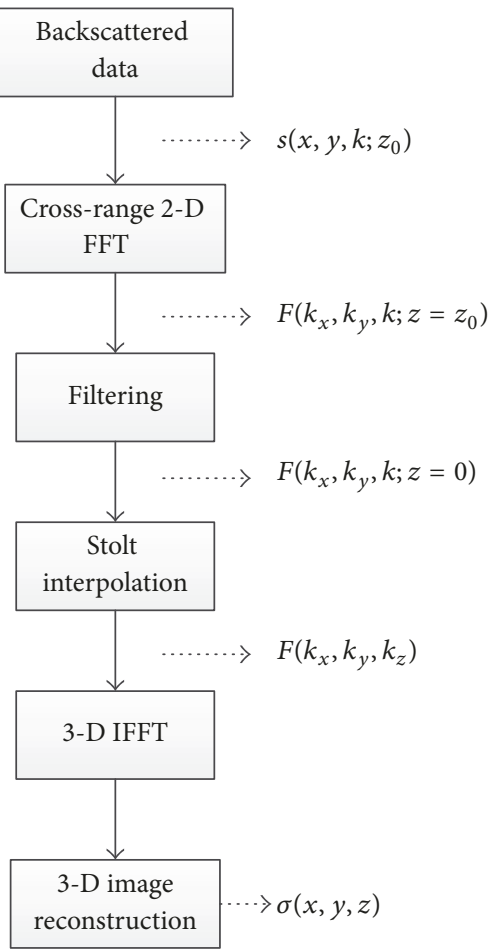

FIGURE 9: MIMO 3D image reconstruction procedure.

Using the proposed MIMO-RMA to reconstruct 3D reflectivity images, the simulation results are shown in Figure 11. The processing time is about $15 \mathrm{~s}$ and the dynamic range is set to $20 \mathrm{~dB}$.

Conventional sampling methods usually take several hours to sample azimuth and elevation angles [20]. Still based on $1 \mathrm{~m} \times 1 \mathrm{~m}$ antenna and $0.5 \mathrm{~m}$ sampling step, the traditional sampling scheme requires 2 hours of processing time. The
TABLE 1: Comparison among different schemes.

\begin{tabular}{lcccccc}
\hline Scheme & (a) & (b) & (c) & (d) & (e) & (f) \\
\hline Dynamic range $(\mathrm{dB})$ & 14 & 3 & 7 & 4.5 & 5 & 8.5 \\
Sampling time $(\mathrm{min})$ & 120 & 2 & 4.1 & 2 & 2 & 4.6 \\
\hline
\end{tabular}

improved sampling scheme can save a lot of time, and six improved sampling schemes are as follows in Figure 12.

In the simulation experiment, we use dynamic range and spatial sampling time as the standard to measure the performance of each sampling scheme.

4.1. Dynamic Range. Figure 13 shows the point scattering model, which contains 10 sets of scattering points with the same reflectance. For the model, the numerical simulation results of the six sampling schemes are shown in Figure 14.

Through the analysis of the results, in addition to Figure $14(\mathrm{~b})$, other sampling programs can produce a corresponding dynamic range of high-resolution images. Scheme (b) lacks the sampling process in the elevation direction, causing some scattering points to be unrecognizable. (a) has a large dynamic range. The actual situation often requires dynamic range conditions of not less than $6 \mathrm{~dB}$, so scheme (c) and scheme (f) can be applied for practical use.

4.2. Spatial Sampling Time. In different operating systems, the spatial sampling time may be different. A typical system consists of a network analyzer that determines the single scan time from the IF bandwidth and the scan point. For example, in the N5247A system, set the IF bandwidth of $10 \mathrm{KHz}$ and set the 401 scanning points; the single scan point of a single scan time is $155 \mathrm{~ms}$ [21]. The dynamic range and sampling processing time of the six sampling schemes are listed in Table 1. 


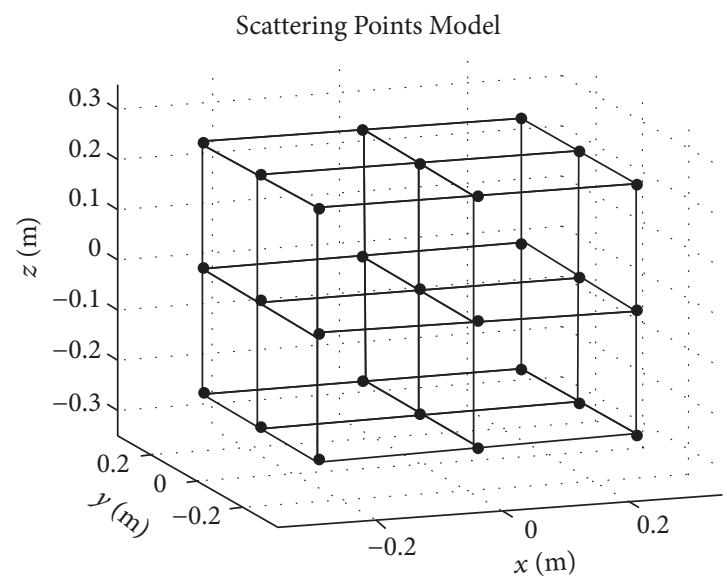

FIGURE 10: Scattering points model.

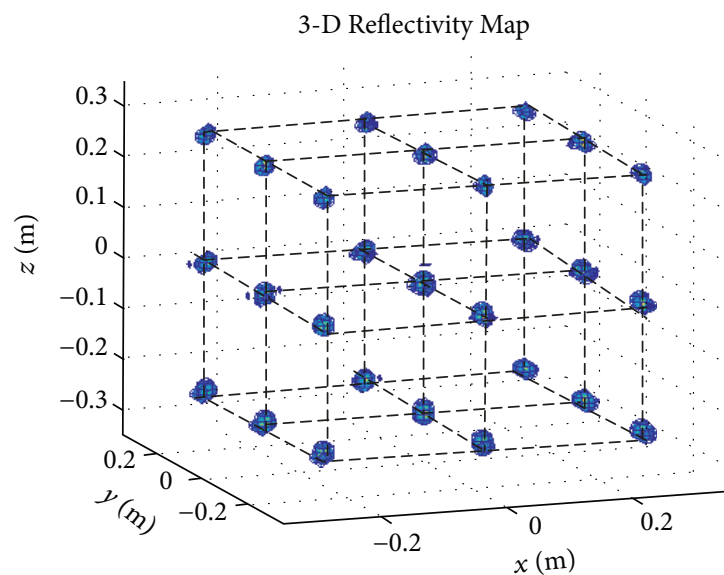

FIGURE 11: 3D reflectivity map.

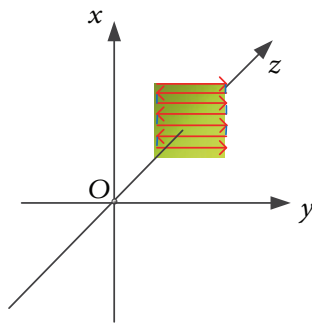

(a)

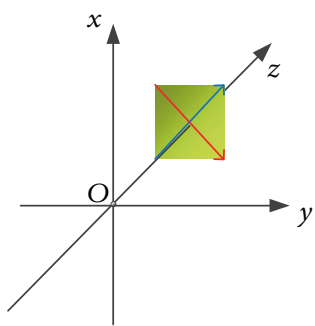

(d)

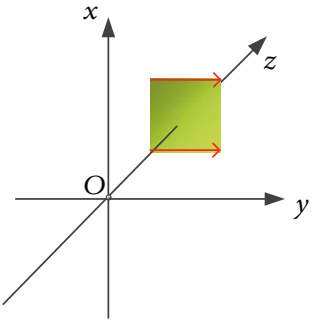

(b)

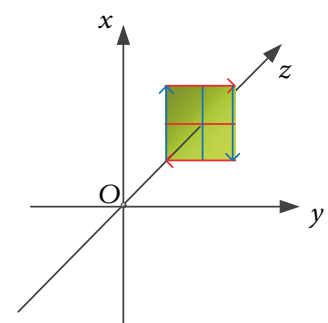

(e)

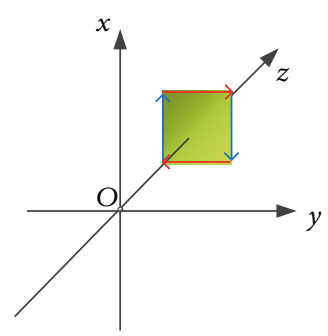

(c)

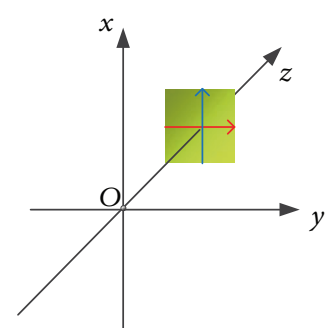

(f)

FIGURE 12: Six spatial sampling schemes. 


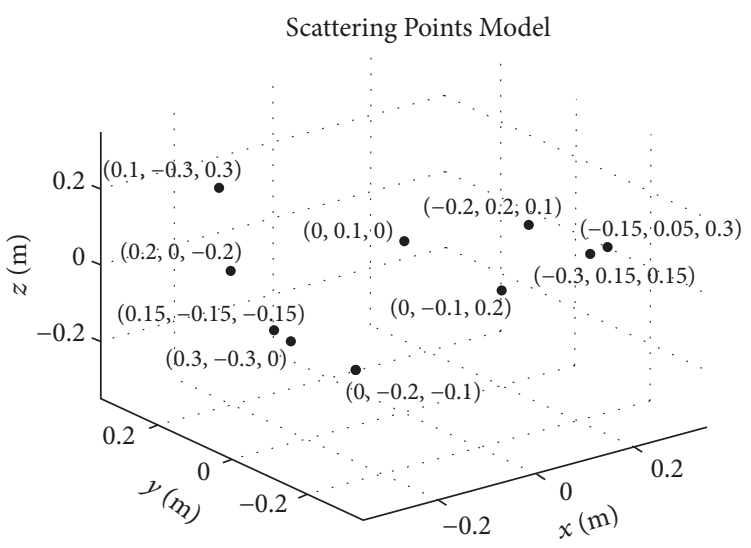

FIGURE 13: Scattering points model.

Scheme (a) with dynamic range $14 \mathrm{~dB}$

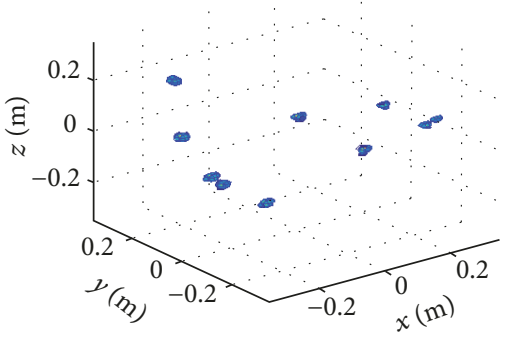

(a)

Scheme (d) with dynamic range $4.5 \mathrm{~dB}$

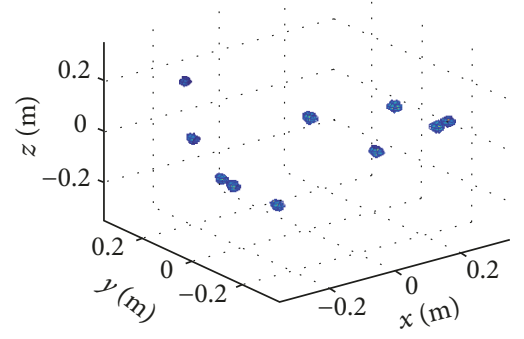

(d)

Scheme (b) with dynamic range $3 \mathrm{~dB}$

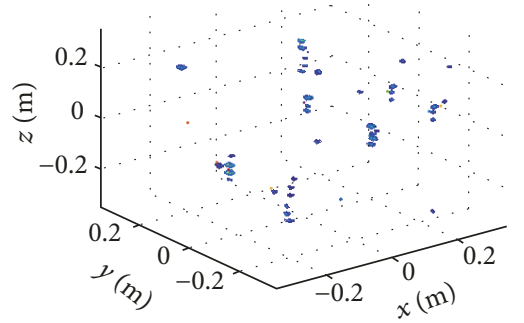

(b)

Scheme (e) with dynamic range $5 \mathrm{~dB}$

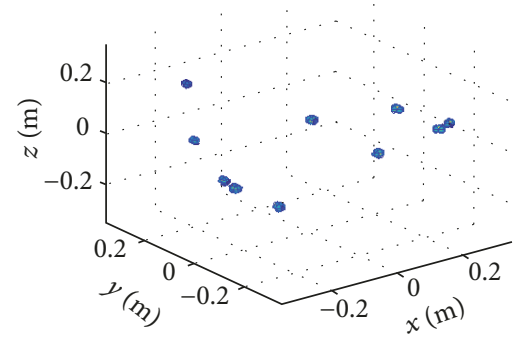

(e)
Scheme (c) with dynamic range $7 \mathrm{~dB}$

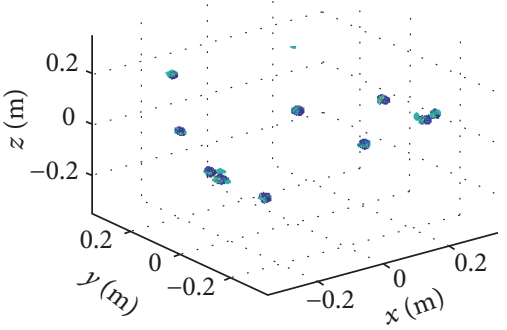

(c)

Scheme (f) with dynamic range $8.5 \mathrm{~dB}$

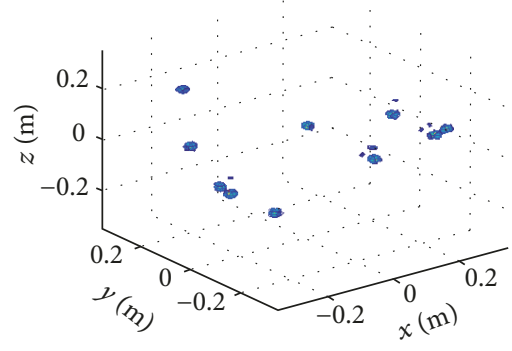

(f)

Figure 14: (a) Scheme (a), 14 dB. (b) Scheme (b), 3 dB. (c) Scheme (c), 7 dB. (d) Scheme (d), 4.5 dB. (e) Scheme (e), 5 dB. (f) Scheme (f), $8.5 \mathrm{~dB}$.

\section{Conclusion}

In this paper, we combine the laboratory measurement of $5 \mathrm{G} R \mathrm{R}$ reflector with the theoretical algorithm to study the application of RF reflector in solving the line-of-sight blocking problem in millimeter-wave frequency band. And a 3D near-field range migration (RM) imaging algorithm for MIMO array configuration is proposed. The algorithm can effectively reduce the image distortion and reconstruct the high-quality image. Finally, by improving the sampling scheme, the sampling time is greatly shortened, which makes the whole algorithm more practical.

\section{Conflicts of Interest}

The authors declare that there are no conflicts of interest regarding the publication of this paper.

\section{References}

[1] L. Wang, S.-W. Qu, J. Li, Q. Chen, Q. Yuan, and K. Sawaya, "Experimental investigation of MIMO performance using passive repeater in multipath environment," IEEE Antennas and Wireless Propagation Letters, vol. 10, pp. 752-755, 2011.

[2] S. S. Yang and K. M. Luk, "Wideband folded-patch antennas fed by L-shaped probe," Microwave and Optical Technology Letters, vol. 45, no. 4, pp. 352-355, 2005.

[3] L. Zheng and D. N. C. Tse, "Diversity and multiplexing: a fundamental tradeoff in multiple-antenna channels," IEEE Transactions on Information Theory, vol. 49, no. 5, pp. 10731096, 2003.

[4] Q. H. Spencer, A. L. Swindlehurst, and M. Haardt, "Zero-forcing methods for downlink spatial multiplexing in multiuser MIMO channels," IEEE Transactions on Signal Processing, vol. 52, no. 2, pp. 461-471, 2004. 
[5] S.-W. Qu, Q.-Y. Chen, Q. Chen, J. Li, Q. Yuan, and K. Sawaya, "Dualantenna system for elimination of blindness in wireless communications," Prog. Electromagn. Res. C, vol. 21, pp. 87-97, 2011.

[6] B. Kim, H. Kim, D. Choi, Y. Lee, W. Hong, and J. Park, " $28 \mathrm{GHz}$ propagation analysis for passive repeaters in NLOS channel environment," in Proceedings of the 9th European Conference on Antennas and Propagation, EuCAP 2015, Lisbon, Portugal, May 2015.

[7] J. Huang and R. J. Pogorzelski, "A ka-band microstrip reflectarray with elements having variable rotation angles," IEEE Transactions on Antennas and Propagation, vol. 46, no. 5, pp. 650-656, 1998.

[8] W. Roh, J.-Y. Seol, J. Park et al., "Millimeter-wave beamforming as an enabling technology for $5 \mathrm{G}$ cellular communications: theoretical feasibility and prototype results," IEEE Communications Magazine, vol. 52, no. 2, pp. 106-113, 2014.

[9] T. Rappaport, S. Sun, R. Mayzus et al., "Millimeter wave mobile communications for 5G cellular: it will work!”, IEEE Access, vol. 1, pp. 335-349, 2013.

[10] G. R. Maccartney, J. Zhang, S. Nie, and T. S. Rappaport, "Path loss models for $5 \mathrm{G}$ millimeter wave propagation channels in urban microcells," in Proceedings of the IEEE Global Communications Conference (GLOBECOM '13), pp. 3948-3953, IEEE, Atlanta, Ga, USA, December 2013.

[11] S. Rajagopal, R. Taori, and S. Abu-Surra, "Self-interference mitigation for in-band mmWave wireless backhaul," in Proceedings of the 2014 IEEE 11th Consumer Communications and Networking Conference, CCNC 2014, pp. 551-556, Las Vegas, NV, USA, January 2014.

[12] J. A. Zhang, S. Hay, and Y. J. Guo, "Directional antennas for point-to-multipoint millimetre wave communications," in Proceedings of the 6th IEEE-APS Topical Conference on Antennas and Propagation in Wireless Communications, IEEE APWC 2016, pp. 204-207, aus, September 2016.

[13] J. G. Andrews, S. Buzzi, and W. Choi, "What will 5G be?" IEEE Journal on Selected Areas in Communications, vol. 32, no. 6, pp. 1065-1082, 2014.

[14] R. Taori and A. Sridharan, "Point-to-multipoint in-band mmwave backhaul for $5 \mathrm{G}$ networks," IEEE Communications Magazine, vol. 53, no. 1, pp. 195-201, 2015.

[15] D. M. Pozar, S. D. Targonski, and H. D. Syrigos, "Design of millimeter wave microstrip reflectarrays," IEEE Transactions on Antennas and Propagation, vol. 45, no. 2, pp. 287-296, 1997.

[16] J. Li and P. Stoica, "MIMO radar with colocated antennas," IEEE Signal Processing Magazine, vol. 24, no. 5, pp. 106-114, 2007.

[17] P. N. Vasileiou, E. D. Thomatos, K. Maliatsos, and A. G. Kanatas, "Adaptive basis patterns computation for electronically steerable passive array radiator antennas," in Proceedings of the 2013 IEEE 77th Vehicular Technology Conference, VTC Spring 2013, Dresden, Germany, June 2013.

[18] J. M. Lopez-Sanchez and J. Fortuny-Guasch, "3D radar imaging using range migration techniques," IEEE Transactions on Antennas and Propagation, vol. 48, no. 5, pp. 728-737, 2000.

[19] A. Ferretti, C. Prati, and F. Rocca, "Permanent scatterers in SAR interferometry," IEEE Transactions on Geoscience and Remote Sensing, vol. 39, no. 1, pp. 8-20, 2001.

[20] N. Li, Y. Zhou, J. Xu, and C. Hu, "A novel method of planar three dimensional synthetic aperture radar imaging," in Proceedings of the 2012 8th International Symposium on Communication Systems, Networks and Digital Signal Processing, CSNDSP 2012, pol, July 2012.
[21] J. Zhao and Z. Dong, "Efficient Sampling Schemes for 3-D ISAR Imaging of Rotating Objects in Compact Antenna Test Range," IEEE Antennas and Wireless Propagation Letters, vol. 15, pp. 650-653, 2016. 


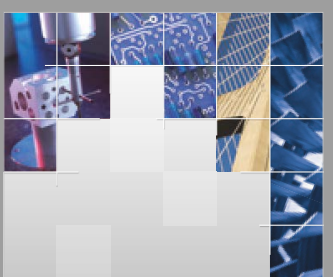

\section{Enfincering}
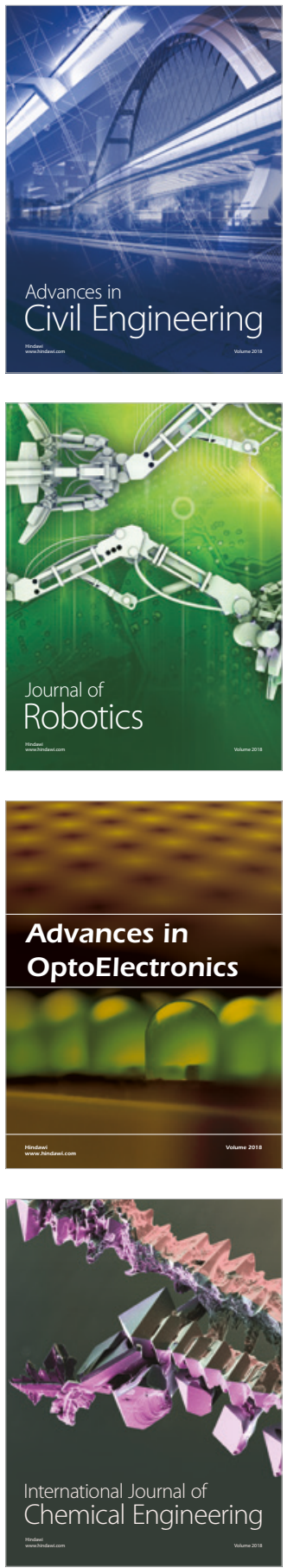

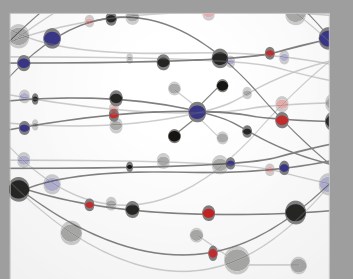

\section{Rotating \\ Machinery}

The Scientific World Journal

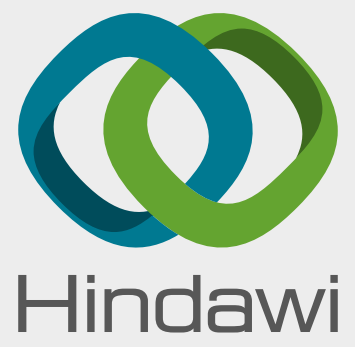

Submit your manuscripts at

www.hindawi.com
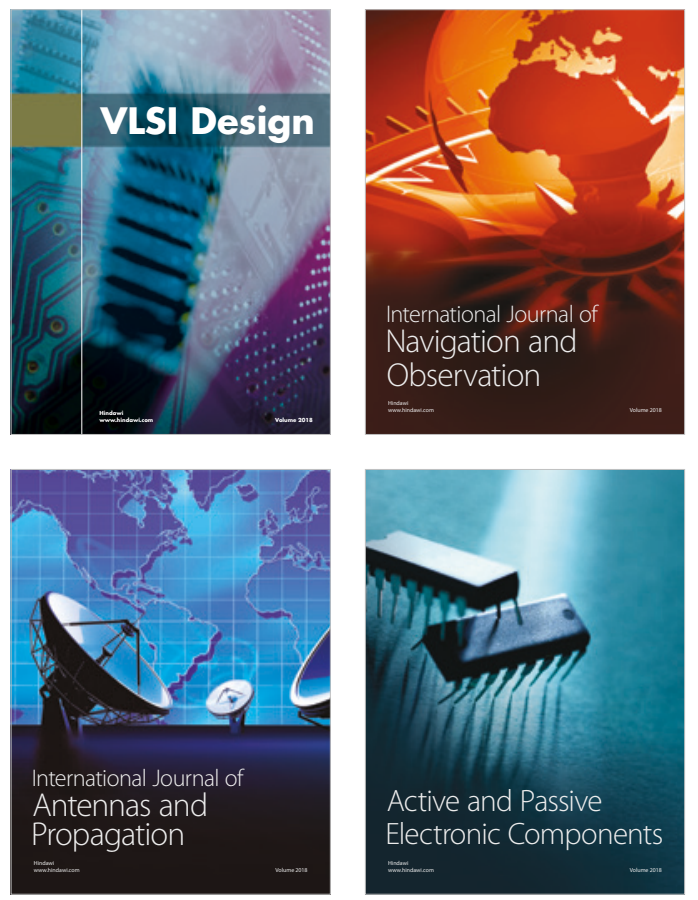
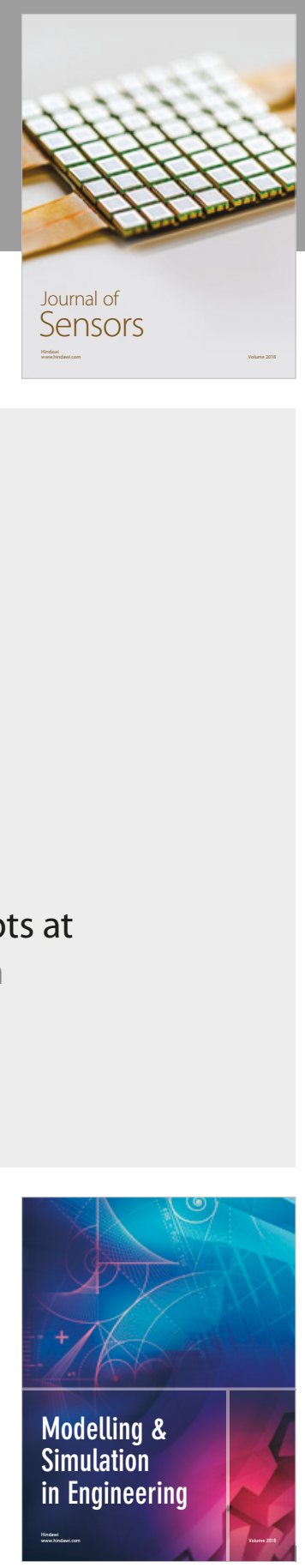

\section{Advances \\ Multimedia}
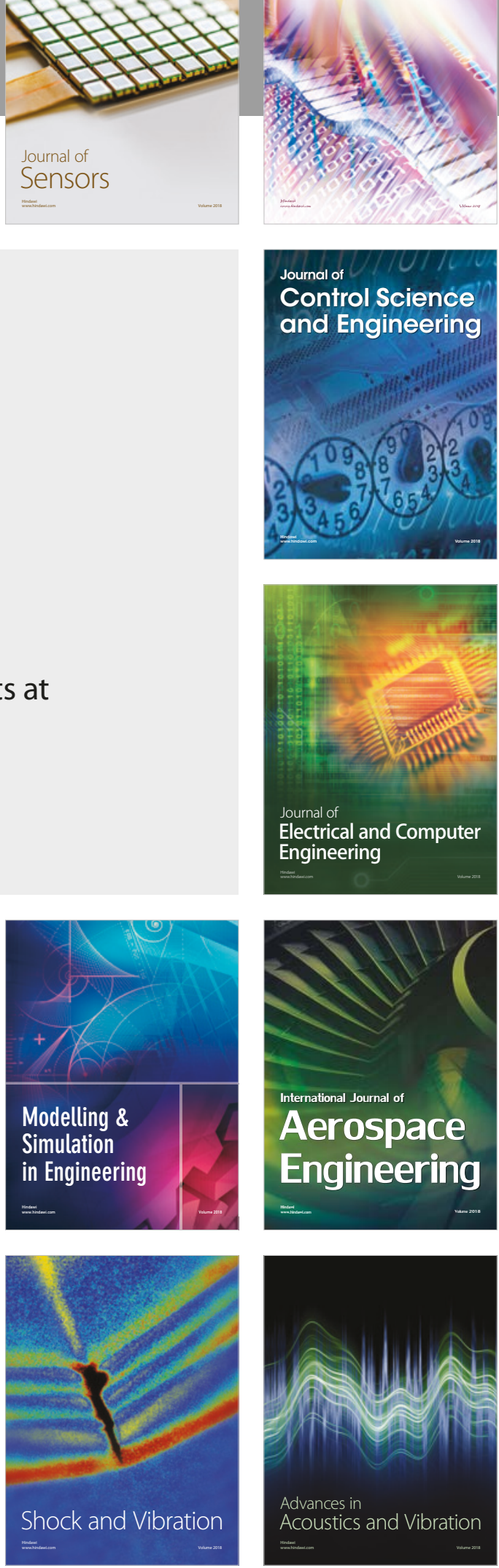